\title{
Western and Mediterranean dietary patterns among Balearic Islands' adolescents: socio-economic and lifestyle determinants
}

\author{
Maria del Mar Bibiloni, Elisa Martínez, Rosa Llull, Antoni Pons and Josep A Tur* \\ Research Group on Community Nutrition and Oxidative Stress, University of Balearic Islands, Guillem Colom \\ Bldg, Campus, E-07122 Palma de Mallorca, Spain
}

Submitted 14 April 2011: Accepted 21 July 2011: First published online 8 September 2011

\begin{abstract}
Objective: To assess prevailing food patterns among Balearic Islands' adolescents, and socio-economic and lifestyle determinants.

Design: Cross-sectional nutritional survey carried out (2007-2008) in the Balearic Islands, a Mediterranean region. Dietary assessment was based on a 145-item semi-quantitative FFQ and two non-consecutive $24 \mathrm{~h}$ recalls. Anthropometric measurements and questions related to socio-economic, lifestyle, physical activity and body image were assessed.

Setting: Data obtained from a representative sample of all inhabitants living in the Balearic Islands aged 12-17 years.

Subjects: A random sample ( $n$ 1231) of the adolescent population (12-17 years old) was interviewed.

Results: Factor analysis identified two major dietary food patterns: 'Western' and 'Mediterranean'. The 'Western' dietary pattern was higher among boys than girls, associated with spending $\geq 4 \mathrm{~h} / \mathrm{d}$ on media screen time, but less prevalent among those adolescents who desired a thinner body and those girls who desired to remain the same weight. The 'Mediterranean' dietary pattern was mainly followed by girls, and also boys who spent $<2 \mathrm{~h} / \mathrm{d}$ on media screen time and girls with high parental socio-economic status.

Conclusions: The present study shows the existence of two major dietary patterns among Balearic Islands' adolescents: 'Western' and 'Mediterranean', but girls are more 'Mediterranean' than boys. This evidence supports that the food pattern of Balearic Islands' adolescents is in a transitional state characterised by the loss of the traditional Mediterranean dietary pattern towards a Western dietary pattern. Low parental socioeconomic status, much leisure-time on sedentary behaviours such as media screen time and body image are factors associated with the 'Western' dietary pattern.
\end{abstract}

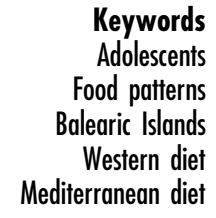

Adolescents

patterns

Western diet

Mediterranean dief
Adolescence is a transitional stage during which many changes take place at physiological and behavioural levels, representing an important life stage for the development of healthy nutrition behaviour ${ }^{(1)}$. Many different factors influence food habits in a complex interactive way ${ }^{(2)}$. Socio-economic and lifestyle factors (parental occupational status, maternal level of education, cultural and/or religious habits, the role of family and patterns of beauty) have a strong influence on eating habits in adolescents ${ }^{(3)}$.

Epidemiological evidence suggests that dietary patterns in the Mediterranean countries are changing rapidly, with an increased consumption of animal products and saturated fat and a decline in intake of basic foodstuffs based on vegetables ${ }^{(4)}$. Recent nutritional surveys carried out in Spain also confirmed a progressive departure from the traditional Mediterranean diet towards a Western dietary pattern, mainly in young generations ${ }^{(5-11)}$. Because there is evidence that nutritional behaviours track from adolescence into adulthood, the promotion of healthy nutrition during adolescence has the potential to confer significant long-term health benefits ${ }^{(1)}$.

Despite the worldwide promotion of the Mediterranean dietary pattern, a progressive shift to a non-Mediterranean pattern could be also developing among Balearic Islands' adolescents. Therefore, the aim of the present study was to assess prevailing food patterns among adolescents living in the Balearic Islands, as well as socio-economic and lifestyle determinants.

\section{Methods}

\section{Study design}

The study is a population-based cross-sectional nutritional survey carried out (2007-2008) in the Balearic Islands, a Mediterranean region. 


\section{Selection of participants, recruitment and approval}

The target population consisted of all inhabitants living in the Balearic Islands aged 12-17 years. The sample population was derived from residents aged $12-17$ years registered in the scholar census of the Balearic Islands. The theoretical sample size was set at 1500 individuals in order to provide a specific relative precision of $5 \%$ (type I error $=0 \cdot 05$; type II error $=0 \cdot 10)$, taking into account an anticipated $70 \%$ participation rate. The sampling technique included stratification according to municipality size, age and sex of inhabitants, and randomisation into subgroups, with Balearic Islands municipalities being the primary sampling units and individuals within the schools of these municipalities comprising the final sampling units. The interviews were performed at the schools. The final sample size was 1231 individuals ( $82 \%$ participation). The main reason for non-participation was the adolescent declined to be interviewed, or the parents did not authorise the interview.

\section{Etbics}

The study was conducted according to the guidelines laid down in the Declaration of Helsinki, and all procedures involving human subjects/patients were approved by the Balearic Islands Ethics Committee. Written informed consent was obtained from all adolescents and their parents or legal guardians.

\section{Dietary assessment}

Dietary questionnaires included two non-consecutive $24 \mathrm{~h}$ diet recall periods, one in the warm season (MaySeptember) and one in the cold season (NovemberMarch) to account for the influence of seasonal variations, and a semi-quantitative FFQ that has previously been validated $^{(12)}$ and applied in other studies and surveys on the Spanish population ${ }^{(9,10,13,14)}$. The FFQ, which asked the participant to recall average use over the past year, consisted of 145 items (118 of the original validated FFQ plus the most characteristic Balearic Islands foods in order to make it easy for the interviewee to answer) arranged by food type and meal pattern. Frequency of food consumption was based on times that food items were consumed (per day, week or month). Consumption $<1$ /month was considered no consumption. The period of consumption of seasonal items was also considered. Edible fractions of foods were recorded in the database. The FFQ foods items were collapsed to twenty-nine food groups (Table 2) that may have practical importance in the daily diet and closely approximated food groups previously reported ${ }^{(15,16)}$. To account for day-to-day intake variability, the questionnaires were administered homogeneously from Monday to Sunday. Well-trained dietitians administered the recalls and verified and quantified the food records.

To estimate volumes and portion sizes, the household measures found in the participants' own homes were used. Conversion of food into nutrients was made using a computer program (ALIMENTA ${ }^{\circledR}$; NUCOX, Palma, Spain) based on Spanish $^{(17,18)}$ and European ${ }^{(19)}$ food composition tables, and complemented with food composition data available for Majorcan food items ${ }^{(20)}$. Identification of misreporters was conducted on the basis of the ratio of energy intake (EI) to BMR. EI:BMR $<0.92$ (boys) and $<0.85$ (girls) was considered to represent underreporting ${ }^{(21)}$, while $\mathrm{EI}: \mathrm{BMR} \geq 2 \cdot 4$ was considered to represent over-reporting ${ }^{(22,23)}$. Under-reporters (20\%) and over-reporters ( $2 \%)$ were excluded from the analysis.

\section{Socio-economic and lifestyle determinants}

A questionnaire incorporating the following questions was used: age group; parental educational level (grouped according to years and type of education: low, $<6$ years; medium, 6-12 years; high, $>12$ years); and parental socio-economic level (based on the occupation of parents and classified as low, medium and high, according to the Spanish Society of Epidemiology $)^{(24)}$. The number of daily meals and snacks was calculated from the total eating occasions that participants declared among the following: breakfast; mid-morning snack; lunch; midafternoon snack; dinner; before going to sleep; others. Three groups of eating frequency were considered: $\leq 3,4$ and $\geq 5$ times $/$ d.

\section{Physical activity patterns}

Physical activity was evaluated according to the guidelines for data processing and analysis of the International Physical Activity Questionnaire (IPAQ) $)^{(25)}$ in the short form and its specific modification for adolescents (IPAQ-A) ${ }^{(26)}$. The specific types of activity assessed were walking, moderateintensity activities (i.e. physical activity at school) and vigorous-intensity activities (i.e. sports practice). In accordance with the AVENA (Food and Assessment of Nutritional Status of Adolescents) study ${ }^{(27)}$, the questionnaire also included information on television (TV) viewing, computer use and video games in $\mathrm{h} / \mathrm{d}$, and usual sleep duration to the nearest 10 min. Physical inactivity was established with a cut-off level of $300 \mathrm{~min} /$ week of moderate/vigorous physical activity, in accordance with current guidelines of physical activity for adolescents ${ }^{(28,29)}$.

\section{Assessment of other covariables}

\section{Anthropometry and adiposity}

Height was determined using a mobile anthropometer (model KaWe 44444; Kirchner \& Wilhelm GmbH Co. KG, Asperg, Germany) to the nearest millimetre, with the subject's head in the Frankfurt plane. Body weight was determined to the nearest $100 \mathrm{~g}$ using a digital scale (model sc9210; Tefal, Rumilly, France). The subjects were weighed in bare feet and light underwear, which was accounted for by subtracting $300 \mathrm{~g}$ from the measured weight. Triceps and subscapular skinfold thickness (ST) 
were measured using a Holtain skinfold calliper (Tanner/ Whitehouse, Crosswell, Crymych, UK) and the mean of three measurements (right arm) was used. Height and weight measures were used to calculate BMI $\left(\mathrm{kg} / \mathrm{m}^{2}\right)$. Body fat percentage (\%BF) was measured from triceps and subscapular ST according to Slaughter et al. ${ }^{(30)}$. This equation has been proposed as the most accurate for estimation of \%BF from ST in this particular population of adolescents $^{(31)}$. \%BF and height were used to calculate fat mass index (FMI; $\mathrm{kg} / \mathrm{m}^{2}$ ).

\section{Defining overweight and obesity}

In children and adolescents, BMI for age has been established as the main measurement to define overweight and obesity $^{(32)}$. However, there are some limitations associated with its use as an indicator of fatness. For example, individuals with increased muscle mass may also have increased $\mathrm{BMI}^{(33)}$. On the other hand, individuals with decreased lean body mass and increased adiposity may also be misclassified by assessment with $\mathrm{BMI}^{(33)}$. Alvero-Cruz et al. ${ }^{(34)}$ showed that the FMI had higher accuracy for overweight screening than BMI. The FMI is a useful measure to evaluate body composition parameters by effectively eliminating differences in body fat associated with height ${ }^{(35)}$. Nevertheless, it is difficult to exclude BMI from the normal-weight and overweight definition. For this reason, a combination of BMI and FMI was used to define overweight and obesity in the present study.

First, subjects were classified using the age- and sexspecific cut-offs developed and proposed for international comparisons by Cole et $\mathrm{al}^{(36)}$, recommended for use also by the International Obesity Taskforce. Then, subjects were classified as normal-fat and overfat according to their FMI using the sex-specific cut-offs proposed by Alvero-Cruz et al. ${ }^{(34)}$ for adolescents: $4.58 \mathrm{~kg} / \mathrm{m}^{2}$ in boys and $7.76 \mathrm{~kg} / \mathrm{m}^{2}$ in girls. Thus, adolescents were classified into two groups as follows: (i) not at risk (BMI for age and sex $<25 \mathrm{~kg} / \mathrm{m}^{2}$ or BMI $\geq 25 \mathrm{~kg} / \mathrm{m}^{2}$ but $\mathrm{FMI}<4.58 \mathrm{~kg} / \mathrm{m}^{2}$ in boys and FMI $<7.76 \mathrm{~kg} / \mathrm{m}^{2}$ in girls); (ii) overweight/obesity (BMI for age and sex $<25 \mathrm{~kg} / \mathrm{m}^{2}$ or $\mathrm{BMI} \geq 25 \mathrm{~kg} / \mathrm{m}^{2}$ but FMI $\geq 4.58 \mathrm{~kg} / \mathrm{m}^{2}$ in boys and FMI $\geq 7.76 \mathrm{~kg} / \mathrm{m}^{2}$ in girls). The variable was labelled as 'body composition'.

\section{Body image}

Self-perceived body image was measured using the Stunkard scale ${ }^{(37)}$, which consists of silhouette drawings ranging from 1 to 9 with monotonic increments in overweight percentage where 1 is the leanest and 9 the heaviest. Separate figures for boys and girls were used. Participants were asked to identify of the nine body figures: 'Which silhouette looks most like yourself?' and 'Which silhouette would you like to look like?' The difference between perceived body image and desired body image was used to determine the level of dissatisfaction with current body image. Values other than zero represent dissatisfaction with perceived body image. A positive value was indicative of the participant's desire to be thinner than his/her perceived current size, while a negative value reflected the participant's desire to be thicker than his/her current perceived size ${ }^{(38,39)}$.

\section{Statistical analyses}

Analyses were performed with the SPSS statistical software package version 19.0 (SPSS Inc., Chicago, IL, USA). Factor analyses by the principal components method and varimax rotation were run on Z-scored transformed food consumption variables in order to identify salient food patterns in the group. To interpret the data, only food groups with factor loading $>0 \cdot 250$ were retained for each factor. For each adolescent, the factor score for each pattern was calculated. These scores were categorised (quintiles), as is often done in dietary epidemiological studies that relate food patterns to health outcomes when there is not a priori knowledge of the function that best fits the data ${ }^{(40)}$. Significant differences in prevalence were calculated by means of the $\chi^{2}$ test. Logistic regression models with the calculations of corresponding adjusted odds ratio and $95 \%$ confidence interval were used to examine differences between adolescents' characteristics and dietary patterns (the fifth quintile $(\mathrm{Q} 5) v$. the first quintile $(\mathrm{Q} 1)$ in each dietary pattern). Univariate analysis was first carried out for all of the socio-economic and lifestyle variables that could be associated with dietary patterns. Any factor was considered a candidate for the multivariate model. Multiple logistic regression analyses were used to simultaneously examine the effect of different socio-economic and lifestyle variables on the dietary patterns. Level of significance for acceptance was $P<0 \cdot 05$.

\section{Results}

Participants' characteristics according to gender are shown in Table 1. Factor analysis retained two major dietary pattern factors which explained $24 \%$ of the total variance: the 'Western' pattern (factor 1, explaining $13.4 \%$ of total variance) and the 'Mediterranean' pattern (factor 2, explaining $10.6 \%$ of total variance; Table 2). Food group categories identified in the 'Western' pattern were yoghurt and cheese, dairy desserts, red meat, poultry, sausages, eggs, bread, cereals, pasta, rice dishes, pizza, fruit juices, canned fruits, nuts, soft drinks, high-fat foods, other oils and fats, sweets and chocolates. Those identified in the 'Mediterranean' pattern included yoghurt and cheese, red meat, poultry, fish and seafood, eggs, legumes, pasta, fresh fruit, fruit juices, vegetables, potatoes and tubercles and olive oil. 'Western' and 'Mediterranean' labels were chosen according to similarities to Western and Mediterranean dietary patterns described elsewhere ${ }^{(41,42)}$. Despite that nuts are a common Mediterranean food ${ }^{(43)}$, in the present study a higher consumption was associated with the 'Western' pattern. However, this association may be 
Table 1 Characteristics of the study population: representative sample of adolescents (aged 12-17 years) living in the Balearic Islands, Spain, 2007-2008

\begin{tabular}{|c|c|c|}
\hline & Boys ( $n$ 574) & Girls (n 657) \\
\hline & $\%$ & $\%$ \\
\hline Age (years) & & \\
\hline $12-13$ & $25 \cdot 8$ & $25 \cdot 6$ \\
\hline $14-15$ & $49 \cdot 3$ & $49 \cdot 6$ \\
\hline $16-17$ & $24 \cdot 9$ & $24 \cdot 8$ \\
\hline Parental educational level & & \\
\hline Low & $32 \cdot 8$ & $36 \cdot 3$ \\
\hline Medium & $29 \cdot 4$ & $32 \cdot 2$ \\
\hline High & $37 \cdot 8$ & $31 \cdot 5$ \\
\hline Parental socio-economic status & & \\
\hline Low & $18 \cdot 4$ & $24 \cdot 3$ \\
\hline Medium & $57 \cdot 6$ & $54 \cdot 8$ \\
\hline High & $24 \cdot 1$ & $20 \cdot 9$ \\
\hline Number of daily meals and sne & & \\
\hline$\leq 3$ & $27 \cdot 7$ & $44 \cdot 1$ \\
\hline 4 & $34 \cdot 9$ & $30 \cdot 7$ \\
\hline$\geq 5$ & $37 \cdot 4$ & $25 \cdot 3$ \\
\hline Media screen time $(\mathrm{h} / \mathrm{d})$ & & \\
\hline$<2$ & $14 \cdot 9$ & $19 \cdot 0$ \\
\hline$\geq 2-<4$ & $43 \cdot 4$ & $38 \cdot 1$ \\
\hline$\geq 4$ & $41 \cdot 7$ & $42 \cdot 9$ \\
\hline Sleep time $(\mathrm{h} / \mathrm{d})$ & & \\
\hline$<7$ & $7 \cdot 5$ & $8 \cdot 2$ \\
\hline$\geq 7$ & $92 \cdot 5$ & $91 \cdot 8$ \\
\hline Physical activity & & \\
\hline Inactive & $22 \cdot 9$ & $47 \cdot 9$ \\
\hline Active & $77 \cdot 1$ & $52 \cdot 1$ \\
\hline Body composition & & \\
\hline Not at risk & $68 \cdot 1$ & $77 \cdot 8$ \\
\hline Overweight/obesity & $31 \cdot 9$ & $22 \cdot 2$ \\
\hline Desire to change weight & & \\
\hline To be thinner & $35 \cdot 9$ & $57 \cdot 7$ \\
\hline To remain the same weight & $37 \cdot 7$ & $35 \cdot 2$ \\
\hline To be thicker & $26 \cdot 5$ & $7 \cdot 1$ \\
\hline
\end{tabular}

associated with the higher consumption of nuts found in the Balearic Islands population than in the overall Spanish population $^{(10)}$.

The 'Western' dietary pattern was found more often among boys (OR $=4 \cdot 47,95 \%$ CI $2 \cdot 81,7 \cdot 11, P<0 \cdot 001)$, while the 'Mediterranean' dietary pattern was mainly followed by girls (OR $=1 \cdot 43,95 \%$ CI $0 \cdot 93,2 \cdot 20$, $P \geq 0 \cdot 05)$. Associations between dietary patterns and socio-economic and lifestyle determinants stratified by gender were assessed (Table 3 for boys and Table 4 for girls). The 'Western' dietary pattern was associated with spending $\geq 4 \mathrm{~h} / \mathrm{d}$ on media screen time in both sexes and with being in the youngest age group (12-13 years) in girls, but was less prevalent among those adolescents who wished to be thinner and those girls who desired to remain the same weight. The 'Mediterranean' dietary pattern was mainly followed by boys who spent $<2 \mathrm{~h} / \mathrm{d}$ on media screen time and girls with low parental socio-economic status. Results also revealed that adolescents who spent $\geq 4 \mathrm{~h} / \mathrm{d}$ on media screen time and were in the highest quintile for the 'Western' dietary pattern showed a higher frequency of consumption of milk (OR $=7 \cdot 86,95 \% \mathrm{CI} 1 \cdot 15$, 53.48; $P<0 \cdot 05)$, soft drinks (OR $=6 \cdot 15,95 \%$ CI 1·23, 30·79;
Table 2 Food patterns identified by factor analyses using the principal components method and varimax rotation: representative sample of adolescents (aged 12-17 years) living in the Balearic Islands, Spain, 2007-2008

\begin{tabular}{|c|c|c|}
\hline \multirow[b]{2}{*}{ Food group category } & \multicolumn{2}{|c|}{ Dietary pattern } \\
\hline & 'Western' & 'Mediterranean' \\
\hline \multicolumn{3}{|l|}{ Dairy food } \\
\hline Milk & - & - \\
\hline Yoghurt and cheese & 0.365 & $0 \cdot 280$ \\
\hline Dairy desserts & 0.628 & - \\
\hline \multicolumn{3}{|c|}{ Meat and processed meats } \\
\hline Red meat & 0.502 & $0 \cdot 291$ \\
\hline Poultry & 0.369 & $0 \cdot 364$ \\
\hline Sausages & 0.440 & - \\
\hline Fish and seafood & - & $0 \cdot 295$ \\
\hline Eggs & 0.382 & 0.416 \\
\hline Legumes & - & 0.450 \\
\hline \multicolumn{3}{|c|}{ Cereals, grains and derivatives } \\
\hline Bread & $0 \cdot 270$ & - \\
\hline Cereals & 0.284 & - \\
\hline Biscuits & - & - \\
\hline Pasta & $0 \cdot 265$ & $0 \cdot 274$ \\
\hline Rice dishes & $0 \cdot 270$ & - \\
\hline Pizza & 0.368 & - \\
\hline \multicolumn{3}{|l|}{ Fruits } \\
\hline Fresh fruits & - & 0.426 \\
\hline Fruit juices & 0.347 & $0 \cdot 277$ \\
\hline Canned fruits & 0.507 & - \\
\hline Vegetables & - & 0.897 \\
\hline Nuts & 0.433 & - \\
\hline Potatoes and tubercles & - & $0 \cdot 897$ \\
\hline \multicolumn{3}{|l|}{ Drinks } \\
\hline Soft drinks & 0.620 & - \\
\hline Tea and coffee & - & - \\
\hline Alcoholic beverages & - & - \\
\hline \multicolumn{3}{|l|}{ Fats } \\
\hline High-fat foods & 0.614 & - \\
\hline Olive oil & - & 0.366 \\
\hline Other oils and fats & 0.388 & - \\
\hline \multicolumn{3}{|l|}{ Sweets and desserts } \\
\hline Sweets & 0.598 & - \\
\hline Chocolates & $0 \cdot 424$ & - \\
\hline
\end{tabular}

Food group factor loadings: only food groups with factor loading $>0.250$ were retained for each factor. Only adolescents who did not misreport their energy intake were considered for this analysis.

$P<0 \cdot 05)$ and nuts (OR $=4 \cdot 01,95 \%$ CI $1 \cdot 02,15 \cdot 80 ; P<0 \cdot 05)$ than their counterparts who spent $<4 \mathrm{~h} / \mathrm{d}$.

In boys, the univariate analysis also showed that the 'Western' dietary pattern was associated with being in the youngest age group (12-13 years), low parental socioeconomic status, spending $\geq 4 \mathrm{~h} / \mathrm{d}$ on media screen time, not being at risk of overweight or obesity and the wish to be thinner; whereas being in the middle age group (14-15 years) and spending $<2 \mathrm{~h} / \mathrm{d}$ on media screen time were associated with the 'Mediterranean' pattern. In girls, low parental educational level, low socio-economic status and spending $\geq 2 \mathrm{~h} / \mathrm{d}$ on media screen time were associated with the 'Western' dietary pattern; whereas medium or high parental educational level, high parental socioeconomic status and spending $<2 \mathrm{~h} / \mathrm{d}$ on media screen time were associated with the 'Mediterranean' pattern. Results also revealed a negative association between age and a desire to maintain the same body shape or a lower 
Table 3 Socio-economic and lifestyle determinants of 'Western' and 'Mediterranean' dietary patterns among adolescent boys, Balearic Islands, Spain, 2007-2008

\begin{tabular}{|c|c|c|c|c|c|c|c|c|c|c|c|c|c|c|}
\hline & \multicolumn{7}{|c|}{ ‘Western' } & \multicolumn{7}{|c|}{ 'Mediterranean' } \\
\hline & \multirow{2}{*}{$\begin{array}{l}\text { Q1 } \\
\%\end{array}$} & \multirow{2}{*}{$\begin{array}{l}\text { Q5 } \\
\%\end{array}$} & \multicolumn{5}{|c|}{ Q1 v. Q5 } & \multirow{2}{*}{$\begin{array}{l}\text { Q1 } \\
\%\end{array}$} & \multirow{2}{*}{$\begin{array}{l}\text { Q5 } \\
\%\end{array}$} & \multicolumn{5}{|c|}{ Q1 v. Q5 } \\
\hline & & & $P$ & Crude ORt & $95 \% \mathrm{Cl}$ & Adjusted OR $\ddagger$ & $95 \% \mathrm{Cl}$ & & & $P$ & Crude ORt & $95 \% \mathrm{Cl}$ & Adjusted OR $\ddagger$ & $95 \% \mathrm{Cl}$ \\
\hline \multicolumn{15}{|l|}{ Age group (years) } \\
\hline $12-13$ & $44 \cdot 4$ & $55 \cdot 6$ & $0 \cdot 108$ & $0 \cdot 36^{\star}$ & $0 \cdot 14,0.98$ & 0.47 & $0 \cdot 12,1 \cdot 80$ & $40 \cdot 5$ & $59 \cdot 5$ & 0.091 & $2 \cdot 59^{*}$ & $1 \cdot 07,6 \cdot 28$ & $2 \cdot 07$ & $0 \cdot 67,6 \cdot 35$ \\
\hline $14-15$ & $29 \cdot 4$ & $70 \cdot 6$ & & $0 \cdot 70$ & $0.28,1 \cdot 73$ & $0 \cdot 75$ & $0.22,2.53$ & $57 \cdot 5$ & $42 \cdot 5$ & & $1 \cdot 30$ & $0 \cdot 61,2 \cdot 77$ & $1 \cdot 24$ & $0.48,3.21$ \\
\hline $16-17$ & $22 \cdot 5$ & $77 \cdot 5$ & & $1 \cdot 00$ & Ref. & $1 \cdot 00$ & Ref. & $63 \cdot 8$ & $36 \cdot 2$ & & $1 \cdot 00$ & Ref. & $1 \cdot 00$ & Ref. \\
\hline \multicolumn{15}{|l|}{ Parental educational level } \\
\hline Low & $31 \cdot 1$ & $68 \cdot 9$ & 0.363 & $1 \cdot 43$ & $0 \cdot 61,3 \cdot 33$ & $1 \cdot 67$ & $0 \cdot 42,6 \cdot 72$ & $65 \cdot 2$ & $34 \cdot 8$ & $0 \cdot 128$ & 0.47 & $0.21,1 \cdot 03$ & 0.49 & $0 \cdot 16,1 \cdot 49$ \\
\hline Medium & $25 \cdot 6$ & $74 \cdot 4$ & & $1 \cdot 88$ & $0 \cdot 77,4 \cdot 55$ & $1 \cdot 77$ & $0.52,6 \cdot 00$ & $60 \cdot 9$ & $39 \cdot 1$ & & 0.56 & $0 \cdot 26,1 \cdot 23$ & 0.93 & $0.33,2.57$ \\
\hline High & $39 \cdot 2$ & $60 \cdot 8$ & & $1 \cdot 00$ & Ref. & $1 \cdot 00$ & Ref. & $46 \cdot 6$ & $53 \cdot 4$ & & $1 \cdot 00$ & Ref. & $1 \cdot 00$ & Ref. \\
\hline \multicolumn{15}{|l|}{ Parental socio-economic status } \\
\hline Low & $13 \cdot 3$ & $86 \cdot 7$ & 0.038 & $4 \cdot 69^{*}$ & $1 \cdot 32,16 \cdot 74$ & $3 \cdot 22$ & $0.56,18 \cdot 45$ & $45 \cdot 7$ & $54 \cdot 3$ & 0.047 & 1.05 & $0 \cdot 40,2 \cdot 74$ & $1 \cdot 32$ & $0.37,4 \cdot 71$ \\
\hline Medium & $35 \cdot 1$ & $64 \cdot 9$ & & $1 \cdot 33$ & $0 \cdot 57,3 \cdot 15$ & $0 \cdot 65$ & $0 \cdot 17,2 \cdot 49$ & $66 \cdot 7$ & $33 \cdot 3$ & & 0.44 & $0 \cdot 19,1 \cdot 02$ & 0.58 & $0 \cdot 20,1 \cdot 71$ \\
\hline High & $41 \cdot 9$ & $58 \cdot 1$ & & 1.00 & Ref. & $1 \cdot 00$ & Ref. & $46 \cdot 9$ & $53 \cdot 1$ & & $1 \cdot 00$ & Ref. & $1 \cdot 00$ & Ref. \\
\hline \multicolumn{15}{|c|}{ Number of daily meals and snacks } \\
\hline$\leq 3$ & $38 \cdot 7$ & $61 \cdot 3$ & 0.347 & 0.53 & $0 \cdot 21,1 \cdot 31$ & 0.93 & $0.25,3.48$ & $63 \cdot 2$ & $36 \cdot 8$ & 0.577 & $0 \cdot 66$ & $0 \cdot 29,1 \cdot 50$ & $0 \cdot 73$ & $0 \cdot 25,2 \cdot 14$ \\
\hline 4 & $33 \cdot 3$ & $66 \cdot 7$ & & $0 \cdot 67$ & $0.29,1.55$ & $1 \cdot 21$ & $0 \cdot 39,3 \cdot 77$ & $53 \cdot 8$ & $46 \cdot 2$ & & 0.97 & $0.47,2.02$ & 0.83 & $0 \cdot 32,2 \cdot 13$ \\
\hline$\geq 5$ & $25 \cdot 0$ & $75 \cdot 0$ & & $1 \cdot 00$ & Ref. & 1.00 & Ref. & $53 \cdot 1$ & $46 \cdot 9$ & & $1 \cdot 00$ & Ref. & $1 \cdot 00$ & Ref. \\
\hline \multicolumn{15}{|l|}{ Media screen time $(\mathrm{h} / \mathrm{d})$} \\
\hline$<2$ & $57 \cdot 7$ & $42 \cdot 3$ & 0.001 & $0 \cdot 15^{\star \star \star}$ & $0 \cdot 05,0.41$ & $0 \cdot 11^{\star *}$ & $0.03,0.47$ & $41 \cdot 2$ & $58 \cdot 8$ & $0 \cdot 011$ & $3 \cdot 05^{*}$ & $1 \cdot 30,7 \cdot 14$ & 3.59 * & $1 \cdot 33,9 \cdot 68$ \\
\hline$\geq 2-<4$ & $34 \cdot 6$ & $65 \cdot 4$ & & $0 \cdot 38^{*}$ & $0.16,0.92$ & $0 \cdot 23^{*}$ & $0.06,0.83$ & $45 \cdot 7$ & $54 \cdot 3$ & & $2 \cdot 54^{*}$ & $1 \cdot 18,5 \cdot 49$ & $2 \cdot 93^{\star}$ & $1 \cdot 11,7 \cdot 71$ \\
\hline$\geq 4$ & $16 \cdot 7$ & $83 \cdot 3$ & & $1 \cdot 00$ & Ref. & $1 \cdot 00$ & Ref. & $68 \cdot 1$ & $31 \cdot 9$ & & $1 \cdot 00$ & Ref. & $1 \cdot 00$ & Ref. \\
\hline \multicolumn{15}{|l|}{ Sleep time $(h / d)$} \\
\hline$<7$ & $16 \cdot 7$ & $83 \cdot 3$ & 0.255 & $2 \cdot 42$ & $0.51,11.51$ & $1 \cdot 73$ & $0 \cdot 17,17 \cdot 32$ & $54 \cdot 5$ & $45 \cdot 5$ & 0.952 & $1 \cdot 04$ & $0.30,3.56$ & $2 \cdot 00$ & $0.30,3.56$ \\
\hline$\geq 7$ & $32 \cdot 6$ & $67 \cdot 4$ & & $1 \cdot 00$ & Ref. & $1 \cdot 00$ & Ref. & $55 \cdot 5$ & $44 \cdot 5$ & & $1 \cdot 00$ & Ref. & $1 \cdot 00$ & Ref. \\
\hline \multicolumn{15}{|l|}{ Physical activity } \\
\hline Inactive & $40 \cdot 9$ & $59 \cdot 1$ & $0 \cdot 103$ & 0.56 & $0.26,1 \cdot 19$ & 0.53 & $0 \cdot 19,1 \cdot 52$ & $65 \cdot 0$ & $35 \cdot 0$ & 0.149 & 0.58 & $0 \cdot 27,1 \cdot 22$ & $1 \cdot 02$ & $0 \cdot 37,2 \cdot 84$ \\
\hline Active & $28 \cdot 0$ & $72 \cdot 0$ & & $1 \cdot 00$ & Ref. & $1 \cdot 00$ & Ref. & $51 \cdot 8$ & $48 \cdot 2$ & & 1.00 & Ref. & $1 \cdot 00$ & Ref. \\
\hline \multicolumn{15}{|l|}{ Body composition } \\
\hline Not at risk & $26 \cdot 4$ & $73 \cdot 6$ & 0.037 & $2 \cdot 26^{*}$ & $1 \cdot 04,4 \cdot 88$ & 0.95 & $0.26,3.50$ & $56 \cdot 8$ & $43 \cdot 2$ & 0.549 & $0 \cdot 80$ & $0 \cdot 39,1 \cdot 66$ & 0.55 & $0.39,1.66$ \\
\hline Overweight/obesity & $44 \cdot 7$ & $55 \cdot 3$ & & $1 \cdot 00$ & Ref. & $1 \cdot 00$ & Ref. & $51 \cdot 3$ & $48 \cdot 7$ & & $1 \cdot 00$ & Ref. & $1 \cdot 00$ & Ref. \\
\hline \multicolumn{15}{|l|}{ Desire to change weight } \\
\hline To be thinner & $57 \cdot 6$ & $42 \cdot 4$ & 0.000 & $0 \cdot 18^{\star \star}$ & $0.06,0.48$ & $0 \cdot 18^{*}$ & $0 \cdot 04,0.86$ & $55 \cdot 6$ & $44 \cdot 4$ & 0.430 & $1 \cdot 38$ & $0 \cdot 60,3 \cdot 15$ & 0.97 & $0.25,3.69$ \\
\hline To remain the same weight & $24 \cdot 1$ & $75 \cdot 9$ & & 0.74 & $0 \cdot 29,1.91$ & 0.88 & $0 \cdot 27,2 \cdot 87$ & $50 \cdot 8$ & $49 \cdot 2$ & & $1 \cdot 67$ & $0 \cdot 77,3 \cdot 61$ & 1.51 & $0.56,4.06$ \\
\hline To be thicker & $19 \cdot 1$ & $80 \cdot 9$ & & $1 \cdot 00$ & Ref. & $1 \cdot 00$ & Ref. & $63 \cdot 3$ & $36 \cdot 7$ & & $1 \cdot 00$ & Ref. & $1 \cdot 00$ & Ref. \\
\hline
\end{tabular}

Q1, first quintile; Q5, fifth quintile; Ref., reference category

Multivariate analysis (multiple logistic regression analysis considering of one explanatory variable). Significant differences between Q5 and Q1 in each dietary pattern $\left(\chi^{2}\right.$ test): ${ }^{\star} P<0.05,{ }^{\star \star} P<0.01,{ }^{\star \star *} P<0.001$. 
Table 4 Socio-economic and lifestyle determinants of 'Western' and 'Mediterranean' dietary patterns among adolescent girls, Balearic Islands, Spain, 2007-2008

\begin{tabular}{|c|c|c|c|c|c|c|c|c|c|c|c|c|c|c|}
\hline & \multicolumn{7}{|c|}{ 'Western' } & \multicolumn{7}{|c|}{ 'Mediterranean' } \\
\hline & \multirow{2}{*}{$\begin{array}{l}\text { Q1 } \\
\%\end{array}$} & \multirow{2}{*}{$\begin{array}{l}\text { Q5 } \\
\%\end{array}$} & \multicolumn{5}{|c|}{ Q1 v. Q5 } & \multirow{2}{*}{$\begin{array}{l}\text { Q1 } \\
\%\end{array}$} & \multirow{2}{*}{$\begin{array}{l}\text { Q5 } \\
\%\end{array}$} & \multirow[b]{2}{*}{$P$} & \multicolumn{4}{|c|}{ Q1 v. Q5 } \\
\hline & & & $P$ & Crude ORt & $95 \% \mathrm{Cl}$ & Adjusted OR & $95 \% \mathrm{Cl}$ & & & & Crude ORt & $95 \% \mathrm{Cl}$ & Adjusted OR & $95 \% \mathrm{Cl}$ \\
\hline \multicolumn{15}{|l|}{ Age group (years) } \\
\hline $12-13$ & $51 \cdot 3$ & $48 \cdot 7$ & $0 \cdot 005$ & $5 \cdot 16^{\star \star}$ & $1 \cdot 86,14 \cdot 33$ & $4 \cdot 30^{\star}$ & $1 \cdot 13,16 \cdot 37$ & $43 \cdot 5$ & $56 \cdot 5$ & 0.679 & 0.96 & $0 \cdot 41,2 \cdot 26$ & $1 \cdot 36$ & $0 \cdot 42,4 \cdot 41$ \\
\hline $14-15$ & $65 \cdot 4$ & $34 \cdot 6$ & & $2 \cdot 87^{\star}$ & $1 \cdot 17,7 \cdot 05$ & $2 \cdot 77$ & $0 \cdot 89,8 \cdot 55$ & $49 \cdot 5$ & $50 \cdot 5$ & & $0 \cdot 76$ & $0.36,1.59$ & $1 \cdot 15$ & $0.45,2.92$ \\
\hline $16-17$ & $84 \cdot 4$ & $15 \cdot 6$ & & $1 \cdot 00$ & Ref. & $1 \cdot 00$ & Ref. & $42 \cdot 5$ & $57 \cdot 5$ & & $1 \cdot 00$ & Ref. & $1 \cdot 00$ & Ref. \\
\hline \multicolumn{15}{|l|}{ Parental educational level } \\
\hline Low & $54 \cdot 1$ & $45 \cdot 9$ & 0.038 & $2 \cdot 44^{*}$ & $1 \cdot 14,5 \cdot 22$ & 0.90 & $0.27,3.03$ & $59 \cdot 3$ & $40 \cdot 7$ & 0.005 & $0.28^{\star \star}$ & $0 \cdot 13,0 \cdot 61$ & 0.65 & $0.21,2.03$ \\
\hline Medium & $71 \cdot 4$ & $28 \cdot 6$ & & $1 \cdot 15$ & $0.52,2.53$ & 0.45 & $0 \cdot 14,1 \cdot 43$ & $46 \cdot 7$ & $53 \cdot 3$ & & 0.47 & $0.22,1 \cdot 02$ & $0 \cdot 70$ & $0.25,1.99$ \\
\hline High & $74 \cdot 2$ & $25 \cdot 8$ & & $1 \cdot 00$ & Ref. & $1 \cdot 00$ & Ref. & $29 \cdot 1$ & $70 \cdot 9$ & & $1 \cdot 00$ & Ref. & $1 \cdot 00$ & Ref. \\
\hline \multicolumn{15}{|l|}{ Parental socio-economic status } \\
\hline Low & $50 \cdot 0$ & $50 \cdot 0$ & 0.005 & $4 \cdot 50^{\star *}$ & $1 \cdot 72,11 \cdot 75$ & $3 \cdot 75$ & $0 \cdot 93,15 \cdot 17$ & $66 \cdot 7$ & $33 \cdot 3$ & 0.000 & $0 \cdot 14^{\star \star \star}$ & $0 \cdot 05,0.40$ & $0 \cdot 13^{\star *}$ & $0.03,0.54$ \\
\hline Medium & $69 \cdot 7$ & $30 \cdot 3$ & & 1.96 & $0 \cdot 81,4 \cdot 77$ & $2 \cdot 15$ & $0 \cdot 68,6 \cdot 82$ & $42 \cdot 1$ & $57 \cdot 9$ & & $0 \cdot 39^{\star}$ & $0 \cdot 15,0.98$ & 0.32 & $0 \cdot 10,1 \cdot 08$ \\
\hline High & $81 \cdot 8$ & $18 \cdot 2$ & & 1.00 & Ref. & $1 \cdot 00$ & Ref. & $21 \cdot 9$ & $78 \cdot 1$ & & $1 \cdot 00$ & Ref. & 1.00 & Ref. \\
\hline \multicolumn{15}{|c|}{ Number of daily meals and snacks } \\
\hline$\leqslant 3$ & $67 \cdot 5$ & $32 \cdot 5$ & 0.004 & $0.46^{\star}$ & $0.22,0.97$ & 0.58 & $0.21,1 \cdot 56$ & $46 \cdot 8$ & $53 \cdot 2$ & $0 \cdot 837$ & $0 \cdot 84$ & $0 \cdot 41,1 \cdot 74$ & 0.49 & $0 \cdot 18,1 \cdot 33$ \\
\hline 4 & $79 \cdot 0$ & $21 \cdot 0$ & & $0 \cdot 25^{\star *}$ & $0.11,0.59$ & $0 \cdot 40$ & $0 \cdot 14,1 \cdot 15$ & $48 \cdot 2$ & $51 \cdot 8$ & & $0 \cdot 80$ & $0.37,1.74$ & 0.69 & $0.25,1.89$ \\
\hline$\geqslant 5$ & $48 \cdot 9$ & $51 \cdot 1$ & & $1 \cdot 00$ & Ref. & $1 \cdot 00$ & Ref. & $42 \cdot 6$ & $57 \cdot 4$ & & $1 \cdot 00$ & Ref. & $1 \cdot 00$ & Ref. \\
\hline \multicolumn{15}{|l|}{ Media screen time $(\mathrm{h} / \mathrm{d})$} \\
\hline$<2$ & $82 \cdot 4$ & $17 \cdot 6$ & $0 \cdot 028$ & $0 \cdot 29^{*}$ & $0.11,0.78$ & $0 \cdot 23^{*}$ & $0.05,0.99$ & $28 \cdot 1$ & 71.9 & 0.062 & $2 \cdot 76^{*}$ & $1 \cdot 13,6 \cdot 73$ & $1 \cdot 82$ & $0 \cdot 62,5 \cdot 40$ \\
\hline$\geqslant 2-<4$ & $70 \cdot 5$ & $29 \cdot 5$ & & 0.56 & $0 \cdot 28,1 \cdot 10$ & 0.51 & $0 \cdot 22,1 \cdot 18$ & $50 \cdot 0$ & $50 \cdot 0$ & & $1 \cdot 08$ & $0.56,2.09$ & 0.85 & $0.38,1.90$ \\
\hline$\geqslant 4$ & $57 \cdot 1$ & $42 \cdot 9$ & & 1.00 & Ref. & 1.00 & Ref. & 51.9 & $48 \cdot 1$ & & $1 \cdot 00$ & Ref. & $1 \cdot 00$ & Ref. \\
\hline \multicolumn{15}{|l|}{ Sleep time $(h / d)$} \\
\hline$<7$ & $71 \cdot 4$ & $28 \cdot 6$ & $0 \cdot 715$ & $0 \cdot 80$ & $0 \cdot 24,2 \cdot 66$ & 0.99 & $0 \cdot 21,4 \cdot 69$ & $41 \cdot 7$ & $58 \cdot 3$ & $0 \cdot 731$ & $1 \cdot 23$ & $0.38,4.03$ & $1 \cdot 03$ & $0.26,3.99$ \\
\hline$\geqslant 7$ & $66 \cdot 7$ & $33 \cdot 3$ & & $1 \cdot 00$ & Ref. & $1 \cdot 00$ & Ref. & $46 \cdot 8$ & $53 \cdot 2$ & & $1 \cdot 00$ & Ref. & $1 \cdot 00$ & Ref. \\
\hline \multicolumn{15}{|l|}{ Physical activity } \\
\hline Inactive & $67 \cdot 8$ & $32 \cdot 2$ & 0.943 & 0.98 & $0.53,1 \cdot 80$ & 0.90 & $0.39,2 \cdot 06$ & $51 \cdot 6$ & $48 \cdot 4$ & $0 \cdot 132$ & 0.63 & $0 \cdot 35,1 \cdot 15$ & 0.69 & $0.31,1 \cdot 50$ \\
\hline Active & $67 \cdot 3$ & $32 \cdot 7$ & & $1 \cdot 00$ & Ref. & $1 \cdot 00$ & Ref. & $40 \cdot 2$ & $59 \cdot 8$ & & $1 \cdot 00$ & Ref. & $1 \cdot 00$ & Ref. \\
\hline \multicolumn{15}{|l|}{ Body composition } \\
\hline Not at risk & $65 \cdot 6$ & $34 \cdot 4$ & $0 \cdot 278$ & 1.57 & $0.69,3.59$ & $1 \cdot 41$ & $0 \cdot 45,4 \cdot 38$ & $48 \cdot 7$ & $51 \cdot 3$ & 0.205 & 0.59 & $0 \cdot 25,1 \cdot 35$ & 0.34 & $0 \cdot 12,1 \cdot 01$ \\
\hline Overweight/obesity & $75 \cdot 0$ & $25 \cdot 0$ & & $1 \cdot 00$ & Ref. & $1 \cdot 00$ & Ref. & $35 \cdot 7$ & $64 \cdot 3$ & & $1 \cdot 00$ & Ref. & $1 \cdot 00$ & Ref. \\
\hline \multicolumn{15}{|l|}{ Desire to change weight } \\
\hline To be thinner & $74 \cdot 5$ & $25 \cdot 5$ & 0.000 & $0 \cdot 08^{\star \star \star}$ & $0 \cdot 02,0.30$ & $0 \cdot 13^{*}$ & $0.03,0.63$ & $45 \cdot 3$ & $54 \cdot 7$ & $0 \cdot 194$ & $3 \cdot 22$ & $0 \cdot 81,12 \cdot 82$ & $3 \cdot 27$ & $0 \cdot 63,16 \cdot 94$ \\
\hline To remain the same weight & $69 \cdot 7$ & $30 \cdot 3$ & & $0 \cdot 10^{\star *}$ & $0.03,0.39$ & $0 \cdot 17^{\star}$ & $0.03,0.82$ & 43.9 & $56 \cdot 1$ & & $3 \cdot 40$ & $0.83,13.98$ & $4 \cdot 44$ & $0 \cdot 87,22 \cdot 80$ \\
\hline To be thicker & $18 \cdot 8$ & $81 \cdot 3$ & & $1 \cdot 00$ & Ref. & 1.00 & Ref. & $72 \cdot 7$ & $27 \cdot 3$ & & $1 \cdot 00$ & Ref. & $1 \cdot 00$ & Ref. \\
\hline
\end{tabular}


one and 'Western' dietary pattern in girls; whereas it was positively related to the 'Mediterranean' dietary pattern (despite the lack of statistical significance). To consume $<5$ daily meals was associated with a low probability to follow a 'Western' dietary pattern among girls, but this variable lost statistical significance after being adjusted for all explanatory variables.

\section{Discussion}

The Mediterranean dietary pattern has been widely reported to be a model of healthy eating for its contribution to favourable health status and best quality of life. However, a progressive shift to a non-Mediterranean pattern, even in countries bordering the Mediterranean Sea, has been observed ${ }^{(44)}$. Previous studies carried out in Spain confirmed that young generations ${ }^{(5-11)}$ are further away from the Mediterranean dietary pattern. Lately, we found that the average adherence to the Mediterranean dietary pattern in our adolescent population was $58 \%{ }^{(11)}$. The present study has identified two major dietary patterns among adolescents ('Western' and 'Mediterranean'), supporting evidence for a nutrition transition from a traditional healthy diet towards a Western diet among Mediterranean youth. The literature has reported that girls paid more attention to foods than boys, met nutritional recommendations, and tried to prevent or reverse the obese state and improve health status ${ }^{(45)}$. Accordingly, we found that girls were more likely to follow the 'Mediterranean' but not the 'Western' dietary pattern than boys.

Socio-economic and lifestyle characteristics are important determinants of the health status in a community. Parental educational level and socio-economic status have a marked effect on children's and adolescents' lifestyles and dietary habits ${ }^{(46)}$. In previous studies, we found that maternal educational level was associated with diet quality $^{(9)}$ and adherence to the Mediterranean dietary pattern $^{(11)}$. Moreover, low socio-economic status and maternal educational level have also been related to high consumption of sweets, high-fat bakery products, sugary and salty snacks ${ }^{(5,47-49)}$. It has also been pointed out that food cost is another factor that may influence people's dietary choices, and to follow a 'Mediterranean' dietary patterns is usually more expensive than following a 'Western' one ${ }^{(50)}$. Our study shows that low parental socio-economic status is strongly associated with the 'Western' pattern, whereas high parental socio-economic status is associated with the 'Mediterranean' pattern.

Children and adolescents usually spend much leisure time on sedentary behaviours, such as TV viewing, computer use and video games, collectively known as screen time ${ }^{(28)}$. An association between watching TV for $>2 \mathrm{~h} / \mathrm{d}$ and major consumption of high-fat snacks and high-sugar drinks has been demonstrated ${ }^{(5,51)}$. TV is clearly related to exposure to the advertising of unhealthy foods ${ }^{(45)}$. However, whereas media screen time was a good proxy for either dietary pattern in the present study, no significant differences were observed when TV and computer use were assessed separately. Thus, adolescents who spent $\geq 4 \mathrm{~h} / \mathrm{d}$ on media screen were more likely to follow a 'Western' dietary pattern. However, those who spent $<2 \mathrm{~h} / \mathrm{d}$ showed the highest probability for a 'Mediterranean' dietary pattern.

Physical activity level has been associated with food choice, and cereals, fruits and vegetables often appear in the diet of active adults and children ${ }^{(52)}$. Children who follow a healthy diet are those who might also maintain high levels of physical activity ${ }^{(53)}$. Despite no significant association between physical activity and the dietary patterns being obtained in the present study, in a previous study we found that sedentary and low-active adolescents showed the lowest adherence to the Mediterranean dietary pattern ${ }^{(11)}$.

On the other hand, no statistically significant association between dietary patterns ('Western' and 'Mediterranean') and sleep time was found in the present study. Taheri ${ }^{(54)}$ suggested that sleep loss leads to more opportunities for food intake. The evidence linking sleep and weight has been stronger for younger children and adults $^{(55,56)}$. In a previous work we found that short sleep $(<7 \mathrm{~h} / \mathrm{d})$ was associated with obesity compared with longer sleep in boys, whereas no association was found in girls ${ }^{(57)}$. Recently Lytle et $a l^{(58)}$ have found more evidence for younger adolescents as compared with older adolescents. Despite that further studies are needed, adequate sleep during this critical time period is important ${ }^{(58)}$.

Despite finding no significant association between number of daily meals and snacks and dietary patterns, the present results indicated that $\geq 5$ eating occasions per day may promote high daily consumption of energy-dense foods and drinks. However, meal pattern and omission of meals, especially skipped breakfast, have also been suggested as markers of an inappropriate dietary intake among adolescents ${ }^{(59)}$, and in a previous study eating frequency was identified as a risk factor for obesity in both boys and $\operatorname{girls}^{(57)}$. Therefore, a promotion of at least 5 daily meals and snacks should be considered in nutrition education programmes for adolescents aimed at reducing risk of disease. However, education programmes could also focus on strategies to promote healthy food choices following the Mediterranean diet.

Our results also revealed that adolescents without risk to be overweight or obese were more likely to follow the 'Western' dietary pattern than the 'Mediterranean' dietary pattern (despite that statistical significance was found only in boys). The Mediterranean diet is an example of a healthy $\operatorname{diet}^{(42)}$. However, controversial results in adherence to the Mediterranean diet and obesity in children and adolescents have been found in the literature. Some studies observed an inverse association between Mediterranean dietary pattern and $\mathrm{BMI}^{(53,60,61)}$, but others found no correlation ${ }^{(62)}$. On the other hand, a study among 2513 Spanish children 
and adolescents aged 10-24 years which assessed adiposity by waist circumference and weight:hip ratio found an inverse association of both indicators with adherence to the Mediterranean diet ${ }^{(63)}$. We have also previously observed that both obese boys and girls avoided sweets and salty snacks consumption to counteract the obesity ${ }^{(57)}$.

Body image has been found to be a powerful determinant of adolescent nutritional habits and food choices ${ }^{(64)}$; and in the present study a desire for a thinner body shape was associated with low risk for the 'Western' dietary pattern. Therefore, understanding how satisfaction with current body shape affects food preferences and the overall adolescent diet is a key issue for the development of strategies aimed at influencing dietary behaviour.

\section{Limitations of the study}

Dietary and physical activity data were based on selfreports ${ }^{(16)}$. The literature reports that food under-reporting is usually associated with gender and weight status ${ }^{(65,66)}$. Self-report of physical activity also can lead to over-report the physical activity due to social desirability bias, and therefore the number of inactive individuals may be greater than that reported ${ }^{(67,68)}$, especially among children and adolescents, and also among the obese ${ }^{(67)}$. However, in many cases, self-reporting is the only feasible method of assessing physical activity ${ }^{(69)}$ and dietary intake in epidemiological studies. Although epidemiologists made every effort to get as accurate data as possible, there is a possibility that misreporting occurred ${ }^{(16)}$.

The FFQ did not differentiate between wholegrain and white bread. However, Ribas-Barba et al. ${ }^{(70)}$ found a slight percentage of daily wholegrain consumers $(14 \cdot 2 \%)$ among the Catalonia population aged $10-75$ years, with a high percentage of never consumers of wholegrain bread $(71 \cdot 3 \%)$. Therefore, we would expect that bread, which was one of the main foods related to the 'Western' dietary pattern in the present study, was consumed mostly as white bread.

The statistical methods usually applied in nutritional epidemiology to define dietary patterns based on collected dietary information contain a posteriori approaches, such as cluster and factor analyses, and a priori dietary index approaches ${ }^{(40)}$. In the present study an exploratory statistical analysis based on factor analyses was chosen. Factor analysis is a useful technique to summarise food patterns and relate them to different socio-economic and lifestyle factors $^{(5)}$ and body self-perception. However, it must be acknowledged that the method is data-specific, thus the patterns and their associations extracted in one study population may not be reproduced in other populations ${ }^{(5,71)}$. This kind of analysis can facilitate the development of interventions aimed at modifying eating patterns, rather than specific components of the diet ${ }^{(5)}$.

Body fat was calculated using Slaughter et al.'s equations (30) which have been suggested previously by Rodríguez et al. ${ }^{(31)}$. However, the present study did not take into account pubertal development despite that chronological age may vary dramatically during this phase. Therefore, as in a previous study ${ }^{(72)}$ in which adolescents have been classified according to their pubertal stage, boys were divided into two groups: pubertal (12-14 years old) and post-pubertal (15-17 years old).

\section{Conclusions}

The present study shows the existence of two major dietary patterns among Balearic Islands' adolescents: 'Western' and 'Mediterranean', but girls are more 'Mediterranean' than boys. This evidence supports that the food pattern of Balearic Islands' adolescents is in a transitional state characterised by the loss of the traditional Mediterranean dietary pattern towards a Western dietary pattern. Low parental socio-economic status, much leisure time on sedentary behaviours such as media screen time and body image are factors associated with the 'Western' dietary pattern.

Adolescents constitute priority targets for action and should be more aware about the Mediterranean diet and its health benefits. Programmes to promote the traditional Mediterranean dietary pattern among not only adolescents but also their families, combined with an active lifestyle, would likely result in a more favourable future health profile.

\section{Acknowledgements}

The study was supported by the Spanish Ministry of Health and Consumption Affairs (Programme of Promotion of Biomedical Research and Health Sciences, Projects 05/1276 and 08/1259; and Red Predimed-RETIC RD06/ 0045/1004) and the Spanish Ministry of Education and Science (FPU Programme, PhD fellowship to M.M.B.). The authors state that there are no conflicts of interest. A.P. and J.A.T. conceived, designed and devised the study; M.M.B., E.M., R.L. and J.A.T. collected and supervised the samples; M.M.B. and J.A.T. analysed the data and wrote the manuscript; A.P. and J.A.T. supervised the study; A.P. and J.A.T. obtained funding.

\section{References}

1. McNaughton SA, Ball K, Mishra GD et al. (2008) Dietary patterns of adolescents and risk of obesity and hypertension. J Nutr 138, 364-370.

2. Aranceta J, Pérez-Rodrigo C, Ribas L et al. (2003) Sociodemographic and lifestyle determinants of food patterns in Spanish children and adolescents: the enKid study. Eur J Clin Nutr 57, Suppl. 1, S40-S44.

3. Neumark-Sztainer D, Hannas PJ, Story M et al. (2003) Family meal patterns: associations with sociodemographic characteristics and improved dietary intake among adolescents. J Am Diet Assoc 103, 317-322.

4. EPIC Group in Spain (2002) Diferencias sociodemográficas en la adhesión al patrón de dieta mediterránea en 
poblaciones de España (Sociodemographic differences in dietary pattern adherence to the Mediterranean diet in Spanish populations). Gac Sanit 16, 214-221.

5. Aranceta J (2001) Spanish food patterns. Public Health Nutr 4, 1399-1402.

6. Moreno LA, Sarriá A \& Popkin BM (2002) The nutrition transition in Spain: a European Mediterranean country. Eur J Clin Nutr 56, 992-1003.

7. Sánchez-Villegas A, Martínez JA, De Irala J et al. (2002) Determinants of the adherence to an 'a priori' defined Mediterranean dietary pattern. Eur J Nutr 41, 249-257.

8. Royo-Bordonada MA, Gorgojo L, de Oya M et al. (2003) Food sources of nutrients in the diet of Spanish children: the Four Provinces study. Br J Nutr 89, 105-114.

9. Tur JA, Romaguera D \& Pons A (2004) Adherence to the Mediterranean dietary pattern among the population of the Balearic Islands. Br J Nutr 92, 341-346.

10. Tur JA, Romaguera D \& Pons A (2004) Food consumption patterns in a Mediterranean region: does the Mediterranean diet still exist? Ann Nutr Metab 48, 193-201.

11. Martínez E, Llull R, Bibiloni MM et al. (2010) Adherence to the Mediterranean dietary pattern among Balearic Islands adolescents. Br J Nutr 103, 1657-1664.

12. Martin-Moreno JM, Boyle P, Gorgojo L et al. (1993) Development and validation of a food frequency questionnaire in Spain. Int J Epidemiol 22, 512-519.

13. Serra-Majem L, Morales D, Domingo C et al. (1994) Comparison of two dietary methods: 24-hour recall and semiquantitative food frequency questionnaire (in Spanish). Med Clin (Barc) 103, 652-656.

14. Bondia-Pons I, Serra-Majem L, Castellote AI et al. (2007) Identification of foods contributing to the dietary lipid profile of a Mediterranean population. Br J Nutr 98, 583-592.

15. Lazarou C, Panagiotakos DB \& Matalas AL (2009) Level of adherence to the Mediterranean diet among children from Cyprus: the CYKIDS study. Public Health Nutr 12, 991-1000.

16. Lazarou C, Panagiotakos DB, Kouta C et al. (2009) Dietary and other lifestyle characteristics of Cypriot school children: results from the nationwide CYKIDS study. BMC Public Health 9, 147.

17. Mataix J, Mañas M, Llopis J et al. (2004) Tablas de composición de alimentos españoles, 4th ed. Granada: INTA-Universidad de Granada.

18. Ortega RM, López AM, Requejo AM et al. (2004) La composición de los alimentos. Herramienta básica para la valoración nutricional. Madrid: Ed. Complutense.

19. Feinberg M, Favier JC \& Ireland-Ripert J (1995) Répertoire géneral des aliments. París: Tec \& Doc Lavoisier.

20. Ripoll L (1992) La cocina de las Islas Baleares, 5th ed. Palma de Mallorca: L. Ripoll Pub. Co.

21. Livingstone MBE \& Black AE (2005) Biomarkers of nutritional exposure and nutritional status. $J$ Nutr $\mathbf{1 3 3}$, 3 Suppl., 895S-920S.

22. Johansson L, Solvoll K, Bjørneboe GA et al. (1998) Underand overreporting of energy intake related to weight status and lifestyle in a nationwide sample. Am J Clin Nutr 68 , 266-274.

23. Mendez MA, Wynter S, Wilks R et al. (2003) Under- and overreporting of energy is related to obesity, lifestyle factors and food group intakes in Jamaican adults. Public Health Nutr 7, 9-19.

24. Alvarez C, Alonso J, Domingo A et al. (1995) La medición de la clase social en ciencias de la salud. Barcelona: SGSociedad Española de Epidemiología.

25. IPAQ - International Physical Activity Questionnaire (2007) Guidelines for data processing and analysis of the International Physical Activity Questionnaire (IPAQ). http://www.ipaq.ki.es (accessed October 2010).

26. Hagströmer M, Bergman P, De Bourdeaudhuij I et al. (2008) Concurrent validity of a modified version of the International
Physical Activity Questionnaire (IPAQ-A) in European adolescents: The HELENA Study. Int J Obes (Lond) 32, 542-548.

27. Vicente-Rodríguez G, Rey-López JP, Martín-Matillas M et al. (2008) Television watching, videogames, and excess of body fat in Spanish adolescents: the AVENA study. Nutrition 24, 654-662.

28. Ferreira CA, Molena CA, Marques RG et al. (2009) Prevalence of physical inactivity and associated factors among adolescents. Rev Assoc Med Bras 55, 523-528.

29. Strong WB, Malina RM \& Blimkie CJR (2005) Evidence based physical activity for school-age youth. J Pediatr 146, 732-737.

30. Slaughter MH, Lohman TG, Boileau RA et al. (1988) Skinfold equations for estimation of body fatness in children and youths. Hum Biol 60, 709-723.

31. Rodríguez G, Moreno LA, Blay MG et al. (2005) Body fat measurement in adolescents: comparison of skinfold thickness equations with dual-energy X-ray absorptiometry. Eur J Clin Nutr 59, 1158-1166.

32. Krebs NF, Himes JH, Jacobson D et al. (2007) Assessment of child and adolescent overweight and obesity. Pediatrics 120, Suppl. 4, S193-S228.

33. Daniels SR (2009) The use of BMI in the clinical setting. Pediatrics 124, Suppl. 1, S35-S41.

34. Alvero-Cruz JR, Alvarez Carnero E, Fernández-García JC et al. (2010) Validity of body mass index and fat mass index as indicators of overweight status in Spanish adolescents: Esccola Study. Med Clin (Barc) 135, 8-14.

35. Baumgartner RN, Koehler KM, Gallagher D et al. (1998) Epidemiology of sarcopenia among the elderly in New Mexico. Am J Epidemiol 147, 755-763.

36. Cole TJ, Bellizzi MC, Flegal KM et al. (2000) Establishing a standard definition for child overweight and obesity worldwide: international survey. BMJ 320, 1240-1243.

37. Stunkard AJ, Sorensen T \& Schulsinger F (1983) Use of the Danish Adoption Register for the study of obesity and thinness. Res Publ Assoc Res Nerv Ment Dis 60, 115-120.

38. Bulik CM, Wade TD, Heath AC et al. (2001) Relating body mass index to figural stimuli: population-based normative data for Caucasians. Int J Obes Relat Metab Disord 25, $1517-1524$.

39. Baptiste-Roberts K, Gary TL, Bone LR et al. (2006) Perceived body image among African Americans with type 2 diabetes. Patient Educ Couns 60, 194-200.

40. Lioret S, Touvier M, Lafay L et al. (2008) Dietary and physical activity patterns in French children are related to overweight and socioeconomic status. J Nutr 138, 101-107.

41. Hu FB, Rimm EB, Stampfer MJ et al. (2000) Prospective study of major dietary patterns and risk of coronary heart disease in men. Am J Clin Nutr 72, 912-921.

42. Trichopoulou A, Costacou T, Christina B et al. (2003) Adherence to a Mediterranean diet and survival in a Greek population. New Engl J Med 348, 2599-2608.

43. Willett WC, Sacks F, Trichopoulou A et al. (1995) Mediterranean diet pyramid: a cultural model for healthy eating. Am J Clin Nutr 61, 6 Suppl., 1402S-1406S.

44. Sofi F, Cesari F, Abbate R et al. (2008) Adherence to Mediterranean diet and health status: meta-analysis. BMJ 337, a1344.

45. Sweeting HN (2008) Gendered dimensions of obesity in childhood and adolescence. Nutr J 7, 1.

46. Fernández PM (2006) Dietary habits and nutritional status of school aged children in Spain. Nutr Hosp 21, 374-378.

47. Lowry R, Kann L, Collins JL et al. (1996) The effect of socioeconomic status on chronic disease risk behaviours among US adolescents. JAMA 276, 792-797.

48. Muñoz KA, Krebs-Smith SM, Ballard-Barbash R et al. (1997) Food intakes of US children and adolescents compared with recommendations. Pediatrics 100, 323-329. 
49. Zive MM, Frank-Spohrer GC, Sallis JF et al. (1998) Determinants of dietary intake in a sample of white and Mexican-American children. J Am Diet Assoc 98, 1282-1289.

50. Lopez CN, Martinez-Gonzalez MA, Sanchez-Villegas A et al. (2009) Costs of Mediterranean and Western dietary patterns in a Spanish cohort and their relationship with prospective weight change. J Epidemiol Community Health 63, 920-927.

51. Rey-López JP, Vicente-Rodríguez G, Répásy J et al. (2011) Food and drink intake during television viewing in adolescents: the Healthy Lifestyle in Europe by Nutrition in Adolescence (HELENA) study. Public Health Nutr 14, 1563-1569.

52. Bellisle F (1999) Food choice, appetite and physical activity. Public Health Nutr 2, 357-361.

53. Lazarou C, Panagiotakos DB \& Matalas AL (2010) Physical activity mediates the protective effect of the Mediterranean diet on children's obesity status: the CYKIDS study. Nutrition 26, 61-67.

54. Taheri S (2006) The link between short sleep duration and obesity: we should recommend more sleep to prevent obesity. Arch Dis Child 91, 881-884.

55. Capuccio FP, Taggart FM, Kandala NB et al. (2008) Metaanalysis of short sleep duration and obesity in children and adults. Sleep 31, 619-626.

56. Patel SR \& Hu FB (2008) Short sleep duration and weight gain: a systematic review. Obesity (Silver Spring) 16, 643-653.

57. Bibiloni MM, Martinez E, Llull R et al. (2010) Prevalence and risk factors for obesity in Balearic Islands adolescents. Br J Nutr 103, 99-106.

58. Lytle LA, Pasch KE \& Farbakhsh K (2011) The relationship between sleep and weight in a sample of adolescents. Obesity (Silver Spring) 19, 324-331.

59. Sjöberg A, Hallberg L, Höglund D et al. (2003) Meal pattern, food choice, nutrient intake and lifestyle factors in The Göteborg Adolescence Study. Eur J Clin Nutr 57, $1569-1578$.

60. Kontogianni MD, Vidra N, Farmaki AE et al. (2008) Adherence rates to the Mediterranean diet are low in a representative sample of Greek children and adolescents. J Nutr 138, 1951-1956.
61. Tsartsali PK, Thompson JL \& Jago R (2009) Increased knowledge predicts greater adherence to the Mediterranean diet in Greek adolescents. Public Health Nutr 12, 208-213.

62. Klesges RC, Klesges LM, Brown G et al. (1987) Validation of the 24-hour dietary recall in preschool children. J Am Diet Assoc 87, 1383-1385.

63. Schröder H, Mendez MA, Ribas-Barba L et al. (2010) Mediterranean diet and waist circumference in a representative national sample of young Spaniards. Int J Pediatr Obes 5, 516-519.

64. Middleman AB \& Duran RH (1998) Eating patterns physical activity and attempts to change weight among adolescents. $J$ Adolesc Health 22, 37-42.

65. Baxter SD, Smith AF, Litaker MS et al. (2006) Body mass index, sex, interview protocol, and children's accuracy for reporting kilocalories observed eaten at school meals. $J \mathrm{Am}$ Diet Assoc 106, 1656-1662.

66. Ventura AK, Loken E, Mitchel DC et al. (2006) Understanding reporting bias in the dietary recall data of 11-yearold girls. Obes Res 14, 1073-1084.

67. Tammelin T, Näyhä S, Laitinen J et al. (2003) Physical activity and social status in adolescence as predictors of physical inactivity in adulthood. Prev Med 37, 375-381.

68. Motl RW, McAuley E \& DiStefano C (2005) Is social desirability associated with self-reported physical activity? Prev Med 40, 735-739.

69. Ekelund U, Neovius M, Linné Y et al. (2005) Associations between physical activity and fat mass in adolescents: the Stockholm Weight Development Study. Am J Clin Nutr 81, 355-360.

70. Ribas-Barba L, Serra-Majem L, Salvador G et al. (2007) Trends in dietary habits and food consumption in Catalonia, Spain (1992-2003). Public Health Nutr 10, 1340-1353.

71. Schulze MB, Hoffman K, Kroke A et al. (2003) An approach to construct simplified measures of dietary patterns from exploratory factor analysis. Br J Nutr 89, 409-419.

72. Alió-Sanz J, Iglesias-Conde C, Pernía JL et al. (2011) Retrospective study of maxilla growth in a Spanish population sample. Med Oral Patol Oral Cir Bucal 16, e271-e277. 